\title{
A 3-Meter Atmospheric Cherenkov Telescope as a Test Bench for Very High Energy Gamma-Ray Astrophysics Projects
}

\author{
T. Yoshikoshi ${ }^{* a}$, R. W. Clay ${ }^{b}$, Y. Matsubara ${ }^{c}$, K. Miyo $^{d}$, M. Mori ${ }^{d}$, T. Naito ${ }^{e}$, \\ K. Nishijima ${ }^{f}$, M. Ohishi $^{a}$, T. Okuda ${ }^{d}$, A. Oshima ${ }^{g}$, G. P. Rowell ${ }^{b}$, T. Saito ${ }^{h}$, T. Sako ${ }^{c}$, \\ H. Tajima ${ }^{c}$, T. Terasawa ${ }^{a}$, T. Yamamoto ${ }^{i}$ \\ ${ }^{a}$ Institute for Cosmic Ray Research, The University of Tokyo, Kashiwa, Chiba 277-8582, Japan \\ ${ }^{b}$ School of Chemistry and Physics, The University of Adelaide, Adelaide, SA 5005, Australia \\ ${ }^{c}$ Solar-Terrestrial Environment Laboratory, Nagoya University, Nagoya, Aichi 464-8602, Japan \\ ${ }^{d}$ Department of Physical Sciences, Ritsumeikan University, Kusatsu, Shiga 525-8577, Japan \\ ${ }^{e}$ Faculty of Management Information, Yamanashi Gakuin University, Kofu, Yamanashi 400-8575, \\ Japan \\ ${ }^{f}$ Department of Physics, Tokai University, Hiratsuka, Kanagawa 259-1292, Japan \\ ${ }^{g}$ Faculty of Engineering, Chubu University, Kasugai, Aichi 487-8501, Japan \\ ${ }^{h}$ Department of Physics, Kyoto University, Kyoto, Kyoto 606-8501, Japan \\ ${ }^{i}$ Department of Physics, Konan University, Kobe, Hyogo 658-8501, Japan \\ E-mail: tyoshikodicrr.u-tokyo.ac.jp
}

\begin{abstract}
We have been setting up a 3-meter diameter atmospheric Cherenkov telescope in Akeno, Japan, for various R \& D studies mainly on very high energy gamma-ray astrophysics. This DaviesCotton type telescope (Akeno telescope, hereafter) was manufactured in 1998 and has been recommissioned at low cost. A low power consumption imaging camera system has been developed for a future gamma-ray astrophysics observatory with "mobile" imaging atmospheric Cherenkov telescopes, which can flexibly change the array configuration for a number of science targets, and has been installed to the Akeno telescope for test observations. We present performances of the optics and the drive system of the Akeno telescope, as well as the status of the imaging camera system. Some other R \& D plans including test observations using a prototype imaging camera for CTA and simultaneous observations of the optical and radio Crab pulsar are also presented.
\end{abstract}

The 34th International Cosmic Ray Conference,

30 July- 6 August, 2015

The Hague, The Netherlands

\footnotetext{
* Speaker.
} 


\section{Introduction}

Ground-based very high energy (VHE) gamma-ray astrophysics has rapidly developed with the atmospheric Cherenkov imaging technique, in which telescopes detect very short (several ns) and faint $\left(\sim 100\right.$ photons $\mathrm{m}^{-2}$ at $\left.\mathrm{TeV}\right)$ Cherenkov light flashes from air showers originated by primary gamma rays at $\mathrm{TeV}$ energies, as resolved images with multi-pixel cameras. The technique has continuously been improved with stereoscopic observations in which the parallax of an air shower is measured simultaneously using multiple telescopes and 3-dimensional development of the shower is reconstructed from the data, so that better angular and energy resolutions are obtained. More than 100 VHE gamma-ray sources have been found [四] since the first source (Crab Nebula) was discovered in 1989 by the Whipple group []]. The next generation ground-based gammaray observatory Cherenkov Telescope Array (CTA) [B] is promoted in collaboration of thousand scientists in the world, to explore the VHE sky further. The project is about to shift to the preproduction phase and reach a critical stage in hardware development, etc.

For imaging atmospheric Cherenkov telescopes (IACTs), two major background components are night sky background (NSB) light and hadronic air showers originated mainly by primary protons. The former consists of airglow, zodiacal light, and star light and is the noise in detecting Cherenkov light images, whereas the latter is the large majority in Cherenkov light images that must be reduced to detect gamma-ray signals with high signal-to-noise ratio. Newly developed hardware components for IACTs, especially imaging cameras, should be tested in the field exposed to these background light. We obtained an old atmospheric Cherenkov telescope which was later reinstalled in Akeno, Japan after recommissioning, as a test bench in the field. This telescope was originally prepared for R \& D studies of TenTen [䧃] or its pathfinder PeV Explorer (PeX), but can also be used in other R \& D. Note also that this is currently the only atmospheric Cherenkov telescope located in Japan and can provide unique opportunity of the field test to scientists developing IACT instruments especially in Japan. We present status of the telescope and ongoing or planned $\mathrm{R} \& \mathrm{D}$ studies with it, as well as recently started collaboration on simultaneous observations of the optical and radio Crab pulsar.

\section{Akeno 3 m Atmospheric Cherenkov Telescope}

The Akeno atmospheric Cherenkov telescope (Akeno telescope) is located at $35^{\circ} 47^{\prime} 12^{\prime \prime} \mathrm{N}$, $138^{\circ} 28^{\prime} 32^{\prime \prime} \mathrm{E}, 902 \mathrm{~m}$ asl, in the Akeno Observatory, Institute for Cosmic Ray Research (ICRR), The University of Tokyo. It has a $\sim 3 \mathrm{~m}$ diameter Davies-Cotton reflector consisting of 18 hexagonal segment mirrors as shown in Figure $\mathbb{W}$. The focal length is also $3 \mathrm{~m}$ and an instrument of up to $\sim 50 \mathrm{~kg}$ in weight can be equipped at the prime focus. When we obtained this old telescope manufactured in 1998, some components had been significantly deteriorated or lost. We reinstalled it at the present position in 2010 after fixing two AC servomotors, repainting the chassis, and so

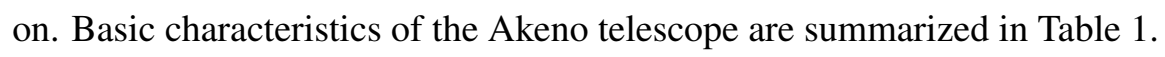

The 18 deteriorated segment mirrors were recoated by ourselves at low cost, using a vacuum deposition system of the Okayama Astrophysical Observatory, National Astronomical Observatory of Japan (NAOJ) [[]]. The mirror reflectivity recovered to $\sim 90 \%$ in the IACT waveband (350$500 \mathrm{~nm}$ ) after that. The recoated segment mirrors were reinstalled to the telescope frame, and 


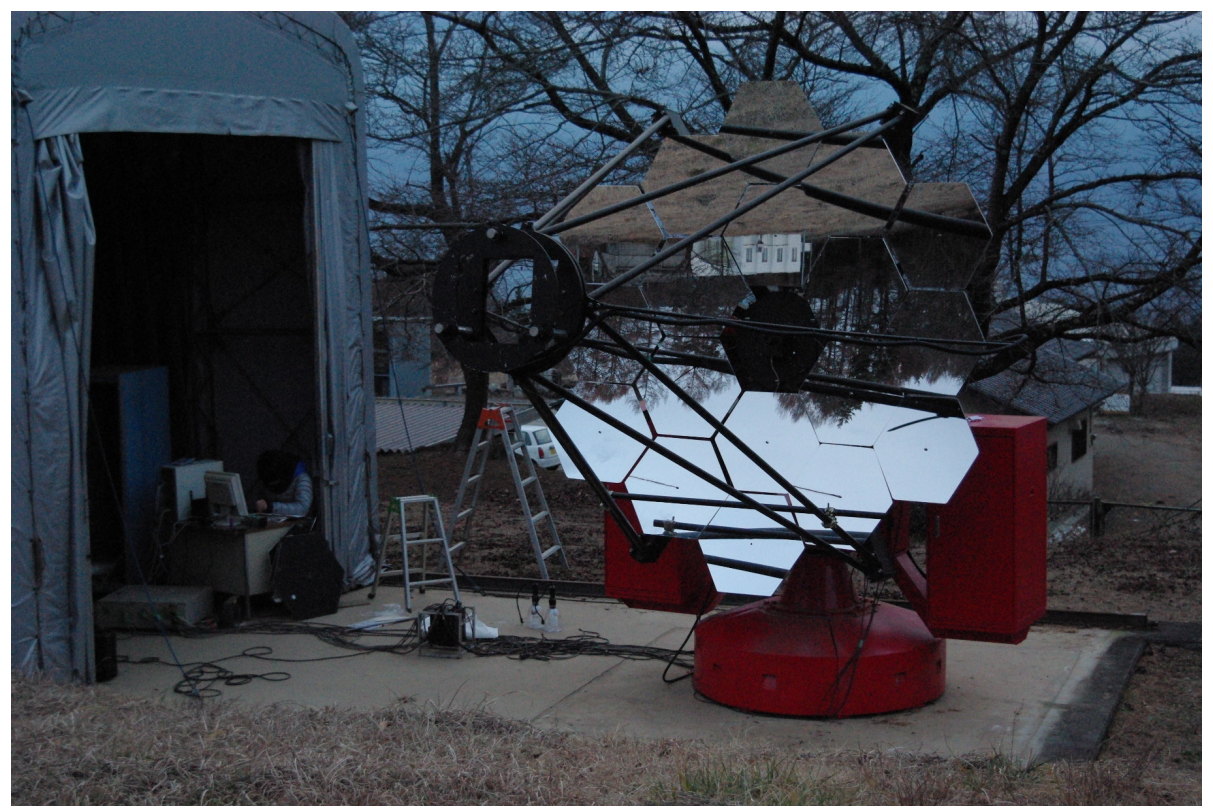

Figure 1: $3 \mathrm{~m}$ atmospheric Cherenkov telescope located in Akeno, Japan.

\begin{tabular}{ll}
\hline Optics & \\
\hline Type & Davies-Cotton \\
Diameter & $\sim 3 \mathrm{~m}$ \\
Focal length & $3 \mathrm{~m}$ \\
Number of segment mirrors & 18 \\
Shape of segment mirrors & Hexagonal \\
Material of mirrors & Glass $(8.5 \mathrm{~mm}$ thickness) \\
Mirror reflectivity & $\sim 90 \%$ \\
Optical point spread function (PSF) & $\lesssim 0.15$ (FWHM) \\
\hline Drive system & \\
\hline Mount & Altazimuth \\
Type of motors & AC servo \\
Encoder resolution & 0.001 \\
Pointing accuracy & $<0.01$ \\
\hline
\end{tabular}

Table 1: Characteristics of the Akeno telescope.

then their directions were manually aligned to form the Davies-Cotton optics. To check the optical performance of the readjusted optics, some bright star images focused on the focal plane were taken by a CCD camera. Figure $\square$ is an image of Vega (visual magnitude 0.03) taken at the elevation angle $44^{\circ}$. The size of the optical point spread function (PSF) is estimated from this result to be $\sigma=3-4 \mathrm{~mm}$, which corresponds to FWHM $\sim 0.15$. This value can possibly be improved further as the star spot in Figure $\square$ seems to have some structure, but for the time being, it is small enough compared to the pixel size (indicated by the black square in the center of Figure D) of the R \& D imaging camera described below. 

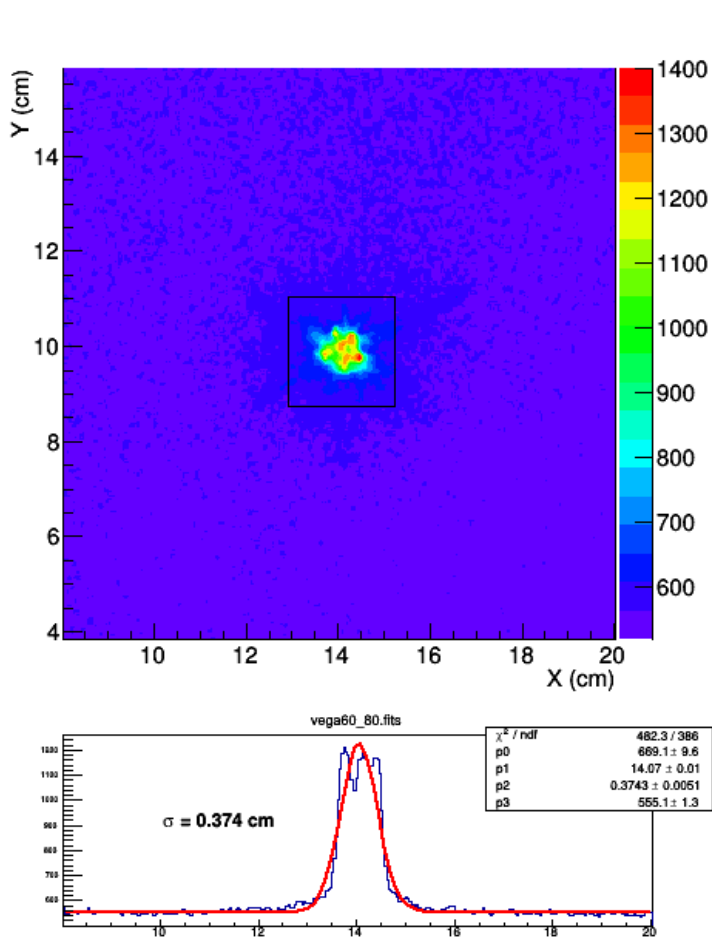

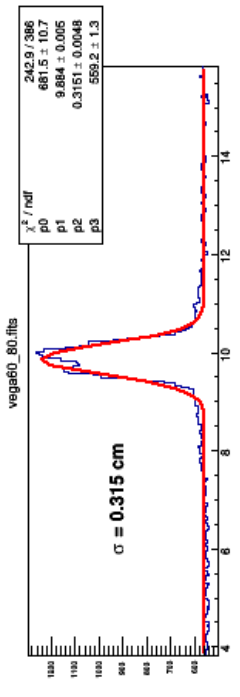

Figure 2: Bright star (Vega) image on the focal plane focused by the readjusted optics of the Akeno telescope. The size of the optical PSF is estimated to be $\sigma=3-4 \mathrm{~mm}$, which is smaller than the pixel size (indicated by the black square in the center) of the $\mathrm{R}$ \& D imaging camera described in Section B.].

The drive system of the Akeno telescope is based on AC servomotors and servo amplifiers. Fortunately, these old components including positioners and encoders can still be used after some refurbishments. However, the computer interface device was lost and we had to look for a suitable one. We successfully found a digital I/O board with PCI interface which can nicely control the positioner from a PC, and then made a computer program in which a simple feedback loop to control the whole drive system is implemented. Some test results show that with this system we can achieve pointing accuracy better than 0.01 which is well smaller than the size of the optical PSF shown above.

\section{Ongoing and Planned R \& D Studies}

\subsection{Low Power Consumption Data Acquisition System for Mobile IACTs}

We have proposed a concept of the "Mobile Telescope Array" project for ground-based gammaray astrophysics, in which the array has a number of movable IACTs and can flexibly change its configuration depending on the science target of interest [ [G]. We can get better sensitivity with a sparse IACT array especially at high energies, whereas a dense IACT array gives better angular and energy resolutions as well as a lower energy threshold. CTA aims to achieve both with IACTs fixed on the ground and therefore the design study using Monte Carlo simulations inevitably becomes a quite large scale $[\square]$. Movable telescopes can be an effective way to optimize the performance of a high energy array for certain circumstances.

To realize mobile IACTs for the future project described above, it is desirable to make movable telescopes run on their own local power supply, independent of cumbersome cables. High 


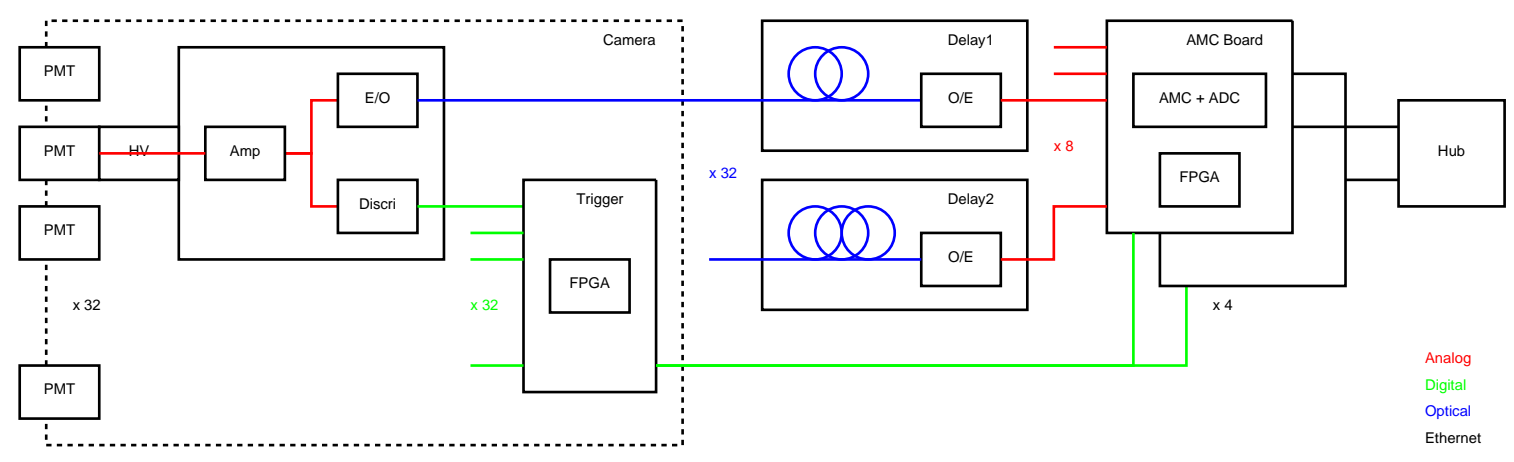

Figure 3: Block diagram of the low power consumption DAQ system.

capacity batteries have very actively been developed recently in many companies and we can expect some batteries fulfilling our requirements being available in the future. Therefore, there is a need to reduce power consumption of the IACT system. We have developed a low power consumption electronics system with an application-specific integrated circuit (ASIC) implementing analog memory cell (AMC) circuits which sample voltages of a Cherenkov signal pulse with a speed of $1 \mathrm{GHz}$. This ASIC chip also has Wilkinson ADCs inside and can further reduce power consumption of the whole circuit. Furthermore, we thought that the sampling depth of $512 \mathrm{~ns}$ (512 cells) of one AMC chip was too long for a single pixel of an IACT as the spread of Cherenkov photon arrival time from a gamma-ray shower is typically several ns, and modified the circuit to share one AMC chip with 8 pixels in combination with optical delays (fibers) of different lengths (64 ns step).

An imaging camera of 32 photomultiplier tubes (PMTs) was also prepared for estimating performance of the above system. The type of the PMTs is Hamamatsu R11265-100 which has a super bialkali photocathode of $23 \mathrm{~mm} \times 23 \mathrm{~mm}$. The preamplified PMT signal is divided to two lines and one of them is fed into an E/O circuit to convert to an analog optical signal, which is transmitted to the data acquisition (DAQ) part by an optical cable. The signal passed through an optical delay is converted back to an electric signal by an O/E unit and finally input to an AMC circuit to record its waveform. The signal of the other line is immediately discriminated and the digital output is sent to a trigger circuit, which examines coincidence of 32 inputs comparing with trigger patterns preset in its FPGA. The block diagram of this system is shown in Figure B. Note that only 4 AMC chips are used in this system to record waveforms from the 32 pixels. We installed these instruments all together to the Akeno telescope in March 2014 as shown in Figure 4.

Figure 5 shows an example of 32 waveforms successfully recorded by the system simultaneously (except one dead pixel in the corner) with a blue LED flashed at the center of the reflector. The waveforms of the same color were recorded by the same AMC chip. We still have some noise problem in discriminators and have not got stable air shower triggers. Test observations of Cherenkov light images from air showers will be done with the Akeno telescope immediately after fixing this issue.

\subsection{Observation of the Optical Crab Pulsar}

The Crab Nebula is known to be the first VHE gamma-ray source and an efficient particle accelerator in our Galaxy. The Crab pulsar is located at the center of the nebula and its spindown 

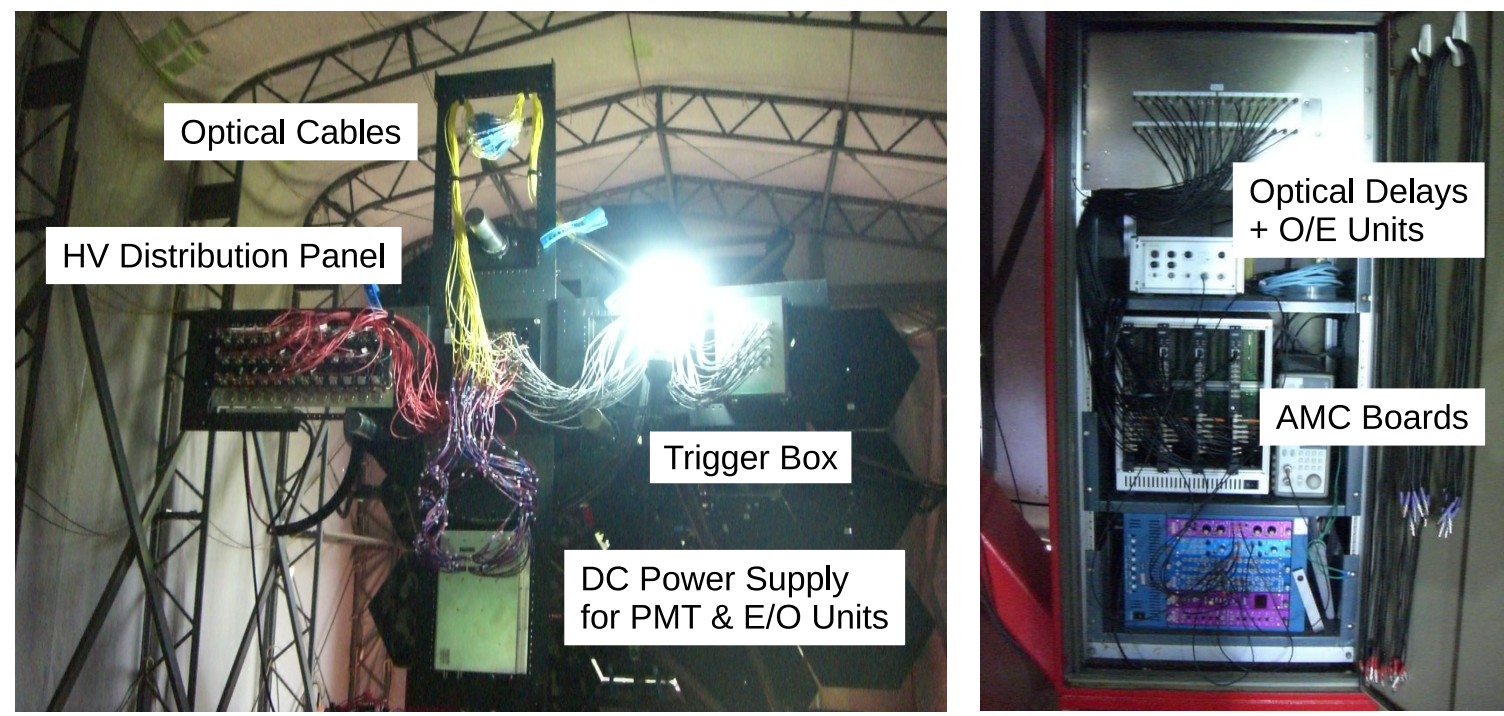

Figure 4: Instruments of the low power consumption DAQ system installed to the Akeno telescope. Left: instruments assembled behind the imaging camera at the focus. The four panels are closed when the telescope is operated. Right: instruments assembled in the 19 inch rack attached to the side of the azimuth chassis.
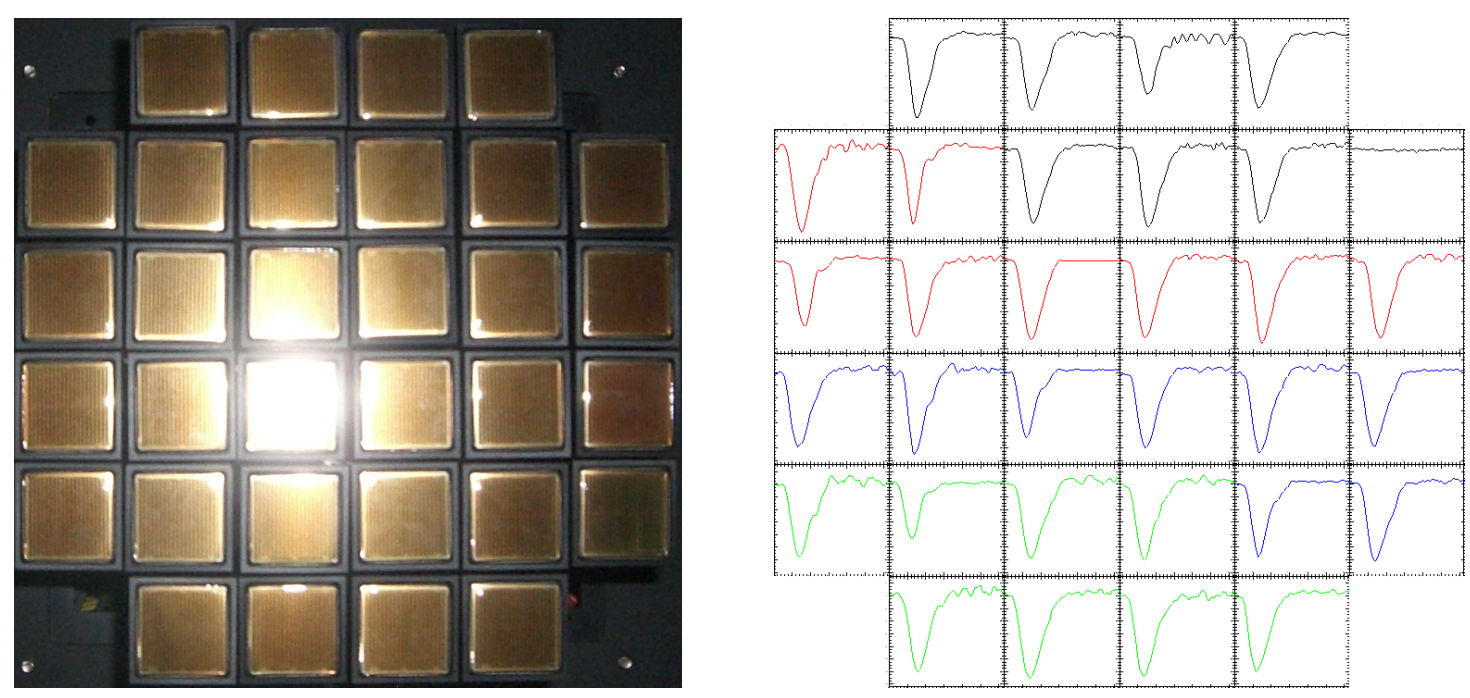

Figure 5: 32 pixel (PMT) imaging camera for the low power consumption DAQ system (left) and $64 \mathrm{~ns}$ waveforms of a blue LED light flash taken by corresponding PMTs (right).

must be the energy source of the nebula. The energy is thought to be transferred to the nebula as relativistic plasma flow (pulsar wind), so that the termination shock of the flow in the inner edge of the nebula can accelerate particles up to above $\mathrm{TeV}$ energies. However, this hypothesis has not been verified enough in a microscopic way.

Radio pulses from the Crab pulsar inevitably pass through the nebula plasma and the effect there in the acceleration site is possibly seen as fluctuation of the dispersion measure (DM). We can utilize the $34 \mathrm{~m}$ radio antenna in Kashima, Japan [ [ $]$ ] which has a good record with precise 


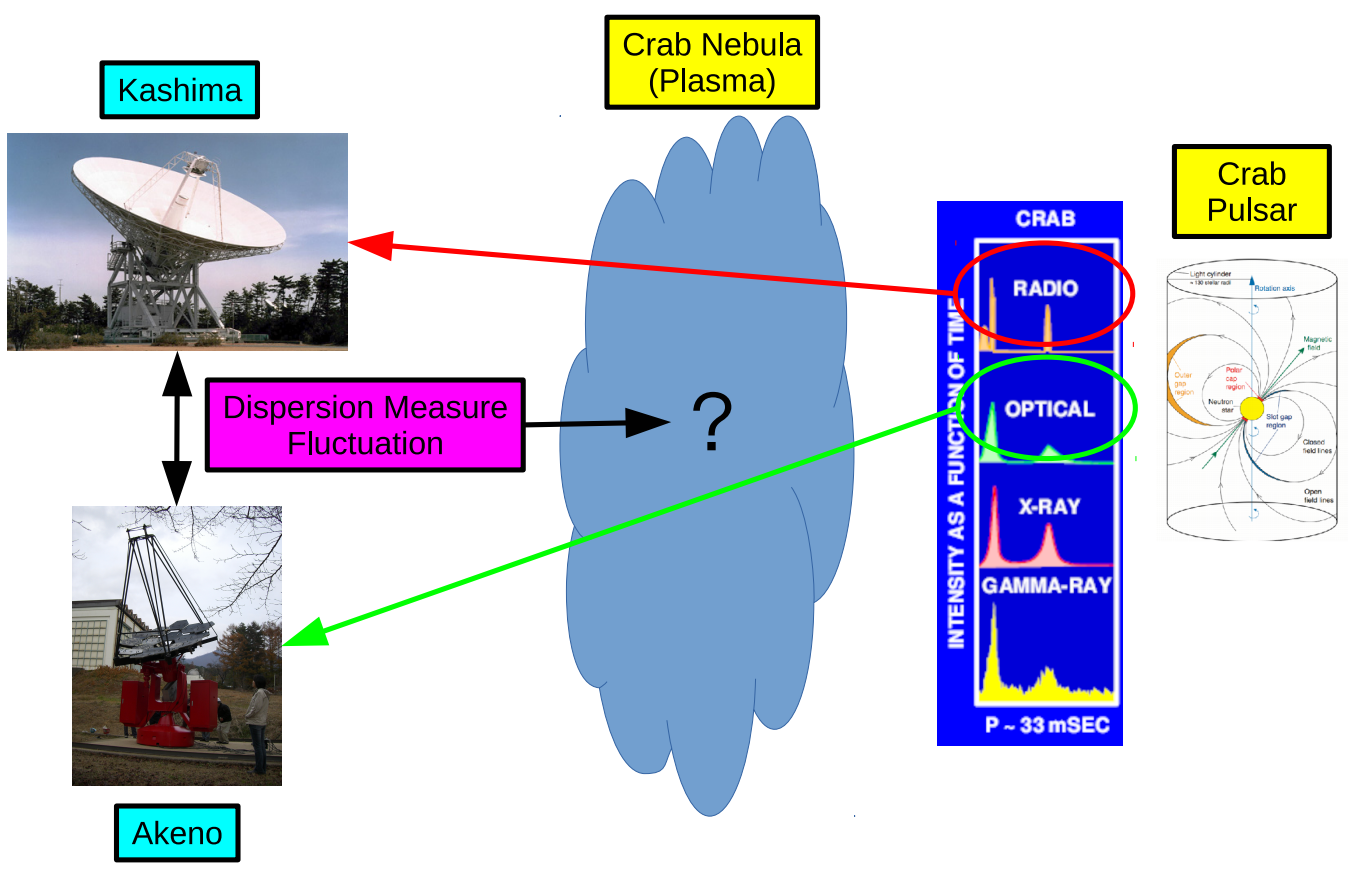

Figure 6: Schematic concept of the new approach observing the Crab Nebula plasma utilizing the radio and optical Crab pulsar.

measurements of the Crab pulsar timing ([Q] for example). However, the DM delay of pulses is dependent on the radio frequency and it is necessary for accurate measurements of DM to give a good reference in another waveband obtained in simultaneous observations. We are motivated for this purpose to observe the optical Crab pulsar using the Akeno telescope which perfectly suits as it is located in the same longitude range as the Kashima antenna and can give more observation time than major optical telescopes in operation. We expect to get new insight on the acceleration site of the Crab Nebula with this new approach. This plan is schematically shown in Figure 6 .

IACTs are suitable to detect the optical Crab pulsar signal owing to large light collecting areas and fast timing responses, and in fact, many successful detections have been reported ([ए]] and references therein). In the Akeno telescope, we plan to use a PMT near the center of the imaging camera described in the previous section, with the photon counting method as demonstrated by the VERITAS group []. We expect to detect statistically significant pulsar signals in a few hours of observation, from the aperture of the Akeno telescope. The project is still in preparation and started with timing calibration of a DAQ PC with a cheap GPS antenna (Garmin GPS 18x), which can receive accurate pulse-per-second (PPS) signals and transmit them to the PC. The clock of the PC is successfully synchronized to UTC with the accuracy of better than $1 \mu \mathrm{s}$, which is sufficient compared to the time scale of the Crab main pulse of $\sim 1 \mathrm{~ms}$.

\subsection{Other R \& D Plans}

Instruments for CTA have actively been developed also in Japan. The CTA-Japan Consortium especially focuses on developing Large-Sized Telescopes (LSTs), which are the largest among three kinds of telescope sizes in CTA. Imaging cameras for LSTs are going to consist of PMTs, 
which can however be replaced by silicon photomultipliers (SiPMs) in the future. There is a plan to test a prototype SiPM camera for LSTs with the Akeno telescope. It is also considered that the CCD camera placed at the center of each LST camera for calibrations will be tested with the Akeno telescope. A compact imaging camera for Small-Sized Telescopes (SSTs) of CTA is developed by the Nagoya group and it will possibly be tested with the Akeno telescope, although this is a backup solution. An R \& D study trying to detect radio emission from air shower particles coincide with the Cherenkov light signal is also planned.

\section{Summary}

We have reinstalled a $3 \mathrm{~m}$ atmospheric Cherenkov telescope in Akeno, Japan, which can be utilized as a test bench in various R \& D studies for future ground-based gamma-ray astrophysics projects, etc. A low power consumption DAQ system was developed for the Mobile Telescope Array concept, and installed to the Akeno telescope for field tests. We confirmed that the system almost worked as we had designed and will perform test observations in the near future to estimate its performance. Some other R \& D studies such as observations of the optical Crab pulsar and field tests of instruments developed for CTA are also ongoing or planned. We are going to keep the quality of the Akeno telescope for future needs in R \& D.

\section{Acknowledgments}

This work was supported by a Grant-in-Aid for Scientific Research (B) of the Japan Society for the Promotion of Science (JSPS) and a Japan-Australia Research Cooperative Program of JSPS and the Australian Research Council (ARC), and is supported by an Inter-University Research Program of ICRR, The University of Tokyo. We thank the Open Source Consortium of Instrumentation (Open-It) lead by the High Energy Accelerator Research Organization (KEK), Japan for the support in the development of AMC.

\section{References}

[1] http://tevcat.uchicago.edu/

[2] T. C. Weekes, et al., Astrophys. J., 342, 379-395 (1989).

[3] https://www.cta-observatory.org/

[4] G. P. Rowell, et al., Nucl. Instrum. Meth. A, 588, 48-51 (2008).

[5] M. Ohishi, et al., Proc. 33rd Internat. Cosmic Ray Conf. (Rio de Janeiro), 587 (2013).

[6] T. Yoshikoshi, et al., Proc. 32nd Internat. Cosmic Ray Conf. (Beijing), 9, 231-234 (2011).

[7] K. Bernlöhr, et al. Astropart. Phys., 43, 171-188 (2013).

[8] M. Sekido, et al., Publ. Astron. Soc. Japan, 51, 595-601 (1999).

[9] R. Mikami, et al., JPS Conf. Proc., 1, 015106 (2014).

[10] J. Hinton, et al., Astropart. Phys., 26, 22-27 (2006).

[11] O. Celik, Ph.D. thesis, University of California, Los Angeles (2008). 